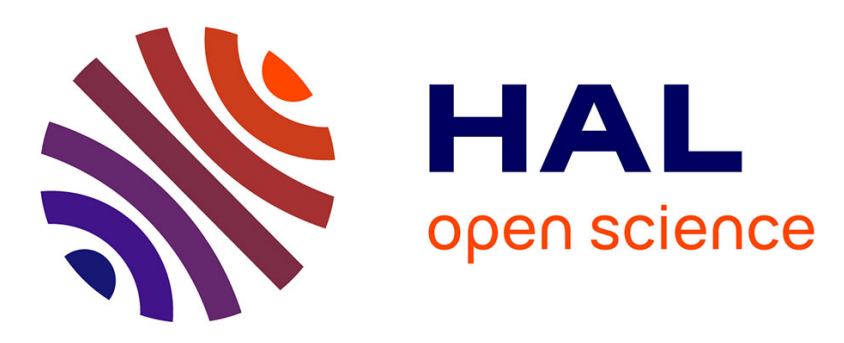

\title{
Polymersomes in "Gelly" Polymersomes: Toward Structural Cell Mimicry
}

Maïté Marguet, Olivier Sandre, Sébastien Lecommandoux

\section{To cite this version:}

Maïté Marguet, Olivier Sandre, Sébastien Lecommandoux. Polymersomes in "Gelly" Polymersomes: Toward Structural Cell Mimicry. Langmuir, 2012, 28 (4), pp.2035-2043. 10.1021/la204018w . hal00744114

\section{HAL Id: hal-00744114 \\ https://hal.science/hal-00744114}

Submitted on 20 Dec 2018

HAL is a multi-disciplinary open access archive for the deposit and dissemination of scientific research documents, whether they are published or not. The documents may come from teaching and research institutions in France or abroad, or from public or private research centers.
L'archive ouverte pluridisciplinaire HAL, est destinée au dépôt et à la diffusion de documents scientifiques de niveau recherche, publiés ou non, émanant des établissements d'enseignement et de recherche français ou étrangers, des laboratoires publics ou privés. 


\title{
Polymersomes in "gelly" Polymersomes: towards
}

\section{structural cell mimicry}

\author{
Maïté Marguet, Olivier Sandre, Sébastien Lecommandoux*
}

Université de Bordeaux/IPB, ENSCBP, 16 avenue Pey Berland, 33607 Pessac Cedex, France. CNRS, Laboratoire de Chimie des Polymères Organiques (UMR5629), Pessac, France.

$$
\text { Fax: (+) } 33540008487
$$

lecommandoux@enscbp.fr

RECEIVED DATE (to be automatically inserted after your manuscript is accepted if required according to the journal that you are submitting your paper to)

TITLE RUNNING HEAD: Biomimetic polymersomes.

CORRESPONDING AUTHOR FOOTNOTE : lecommandoux@enscbp.fr 


\section{ABSTRACT.}

We demonstrate here the formation of compartmentalized polymersomes with an internal «gelly» cavity using an original and versatile process. Nanosize polymersomes of poly(trimethylene carbonate)$b$-poly(L-glutamic acid) PTMC- $b$-PGA, formed by a solvent displacement method are encapsulated with a rough "cytoplasm mimic" in giant polymersomes of poly(butadiene)- $b$-poly(ethylene oxide) PB- $b$ PEO by emulsion-centrifugation. Such a system constitutes a first step towards the challenge of structural cell mimicry with both "organelles" and "cytoplasm mimics". The structure is demonstrated with fluorescence labeling and confocal microscopy imaging with movies featuring the motion of the inner nanosize polymersomes in larger vesicles. Without "cytoplasm mimic", the motion was confirmed to be Brownian by particle tracking analysis. The inner nanosize polymersomes motion was blocked in the presence of alginate, but only hindered in the presence of dextran. With the use of such high molecular weight and concentrated polysaccharides, the crowded internal volume of cells, responsible for the so-called "macromolecular crowding" effect influencing every intracellular macromolecular association, seems to be efficiently mimicked. This study constitutes major progress in the field of structural biomimicry and will certainly enable the rise of new, highly interesting properties in the field of high-added value soft matter. 


\section{Introduction}

Polymer vesicles or polymersomes are resulting from the self-assembly of amphiphilic block copolymers in aqueous media, and are often presented as the structural analogues of liposomes. ${ }^{1,2}$ They can load both hydrophilic (in their internal aqueous reservoir) and hydrophobic components (in their membrane). Their similarity to liposomes is however limited to this closed bilayer structure. Indeed, due to their polymeric nature, polymersomes present an intrinsically thicker membrane, therefore with higher hydrophobic component loading capacity, which confers them a larger mechanical stability and lower permeability compared to liposomes. ${ }^{3}$ These properties, in addition with the inherent chemical versatility of polymer building blocks, are responsible for their considerable interest and development ${ }^{4}$ as drug delivery systems ${ }^{5}$, more particularly stimuli-sensitive carriers, ${ }^{6-8}$ as sensors and nanoreactors ${ }^{9-13}$.

While liposomes mimic the semi-permeable living cell membrane with their intrinsic phospholipid nature, polymersomes' structure and properties are much closer to those of viral capsids. ${ }^{14}$ However, neither liposomes nor polymersomes can be described as cell mimics as an essential key towards cell mimicry lies in compartmentalization. Yet, one could question the relevance of biomimicry and an elegant answer by Feynman was "what I cannot create, I cannot understand". ${ }^{15,16}$ From the most evident and fundamental point of view, artificial mimics as model systems enable to complement biological studies as they allow to dissociate parameters closely correlated in nature. ${ }^{15}$ Moreover, the first step that consists in mimicking the multicompartmentalized structure of a cell is already very challenging. It is only once their formation is controlled and optimized that it becomes possible to take advantage of these mimics to gain new properties with innovative soft materials. As a matter of fact, Nature evolved with compartmentalization because such a structure offers multiple advantages. For artificial compartmentalized cell mimics, one of the most foreseeable benefits obviously lies in a better protection $^{17,} 18$ of inner encapsulated actives, often very fragile in the field of drug delivery. Furthermore, several compartments that each contains a different active in the same structure open a path to combinatory drug delivery. ${ }^{17-19}$ Such an approach is of particular interest in oncology as it 


\section{Langmuir}

enables to load and potentially deliver incompatible drug cocktails together. ${ }^{20,21}$ Going further, while a certain permeability is necessary for drug delivery systems, compartmentalization can also circumvent undesirable prematurely drug leakage especially in liposomes, as demonstrated by Zasadzinski and coworkers. $^{22}$ Indeed, compartmentalized systems can be used to finely tune permeability. ${ }^{17,}{ }^{18,}{ }^{23}$ Such complex structure can also impact the way we design a chemical reaction or induce a reaction only when different components are mixed. ${ }^{24-26}$ Caruso and coworkers in particular pointed out the great level of possibilities arising from microencapsulated reactors, one example being their promising capsosomes. $^{27-29}$

In a perspective of compartmentalization, some very interesting work has already been achieved with liposomes but this field is only in its early stages regarding polymersomes. In the most recent approach, solvent displacement method was used, with the aqueous solution being a suspension of smaller polymersomes previously generated by a film rehydration of a lamellar forming amphiphilic block copolymer. ${ }^{30}$ The main drawback of this method is essentially based on the poor encapsulation yield during the solvent displacement process. To overcome this limitation, the most promising alternative lies in methods based on emulsions or double emulsions. Chiu et al. ${ }^{31}$ were the first to tackle this challenge with polymersomes; however, even though their method consisting of using two successive double emulsions was very original, it may not yield the most reproducible and homogeneous systems while being difficult to use on a daily basis. In order to gain in reproducibility and homogeneity of the preparation, Weitz and coworkers developed highly sophisticated microfluidics devices. In a first step, they were able to control the formation of polymersome aggregates or multicompartment polymersomes ${ }^{20}$ before yielding fully multicompartmentalized polymersomes. ${ }^{21}$ The size of the internal polymersomes is however limited to quite larger dimensions $(\approx 100 \mu \mathrm{m})$, depending on the capillary size. In addition, this method is rather tricky since several issues need to be solved before achieving the high throughput production of polymersomes by microfluidics: possible coalescence of the internal and external aqueous phases, dewetting instability of the organic phase from the copolymer bilayer usually 


\section{Langmuir}

leading to excess polymer patched to the vesicles, ${ }^{32}$ and unequal evaporation rate of solvents from the organic phase during the rather long drying step of the copolymer membranes with dramatic consequences on contact angles. ${ }^{33}$

Regarding cell biomimicry, in addition to compartmentalized vesicles that can mimic the organelles, cytoplasm also plays an important role in cellular activity and regulation. In this respect, vesicles with gelly or gelified cavity, also names "hydrosomes", have been investigated for approximately a decade. Of course, there are again many interesting properties arising when mimicking a viscoelastic cytoplasm like a slower diffusion of actives towards the outside environment and above all a better protection and thus stability and shape integrity, by absorption of mechanical stress. An approach that is often used, in particular by A. Viallat and coworkers, consists in encapsulating a poly( $\mathrm{N}$-isopropylacrylamide) PNIPAAm based solution, which will in situ photopolymerize into covalently cross-linked gels upon UV irradiation. ${ }^{34-41}$ However, in cells, the cytoskeleton is composed of the protein filaments actin, microtubules and intermediate filaments, formed by nucleation-elongation processes. The growth of the protein filaments of this cytoskeleton is driven by non-covalent interactions, making them very dynamic with constant association/dissociation processes. ${ }^{15}$ For that reason, non-covalent or physical gels constitute in our opinion a better alternative. Limozin and Sackmann ${ }^{42}$ were impressively able to form liposomes with dynamic actin networks cross-linked by the natural cross-linkers $\alpha$-actinin and filamin. Other approaches were developed using the LCST character of non-crosslinked PNIPAAm, ${ }^{43,44}$ electrostatic complexation, ${ }^{45-47} \mathrm{pH}$-dependent solubility and thus gelling, ${ }^{48}$ polysaccharide based systems, ${ }^{49}, 50,46,47,51$ or more recently peptide amphiphiles ${ }^{52}$ to gelify the inside reservoir of liposomes . To the best of our knowledge Feijen and coworkers were the first to tackle this aim with polymersomes using PNIPAAm as a gelator. ${ }^{53,54}$

The aim of this work is to go on step further towards structural cell mimicry and to combine both compartmentalized structures (to mimic organelles) and a "gelly" cavity (as cytoplasm mimic) in polymersomes. Such a realization requires an exquisite control of the physical parameters and 


\section{Langmuir}

interaction components, together with an efficient process. The achievement of this original and innovative biomimetic structure constitutes a first necessary step before taking advantage of it to address other challenges in controlled catalysis and chemical (bio)reactions.

\section{Experimental Section}

2.1. Materials and reagents: Poly(butadiene) $)_{30}-b$-poly(ethylene oxide $)_{46}\left(\mathrm{~PB}_{30}-b\right.$ - $\left.\mathrm{PEO}_{46}\right)(\mathrm{P} 9095-\mathrm{BdEO}$, $\mathrm{M}_{\mathrm{n} \text { PB}}=2500 \mathrm{~g} / \mathrm{mol}$ and $\left.\mathrm{M}_{\mathrm{n} \text { PEO}}=1300 \mathrm{~g} / \mathrm{mol}, \mathrm{I}=1.04\right)$ and amino-terminated poly(butadiene $)_{20}\left(\mathrm{~PB}_{20}\right)($ for polymersome membrane labeling) (P3977- $\mathrm{BdNH}_{2}, \mathrm{M}_{\mathrm{n}}=1700 \mathrm{~g} / \mathrm{mol}, \mathrm{I}=1.11, \mathrm{f}>0,98 \%$ ) were purchased from Polymer Source. Alexa Fluor 568-carboxylic acid, succinimidyl ester (A20003-1mg, mixed isomers, $791.8 \mathrm{~g} / \mathrm{mol}$ ) and Alexa Fluor 488 carboxylic acid, succinimidyl ester (A20000-1mg, mixed isomers, $643.41 \mathrm{~g} / \mathrm{mol}$ ) were purchased from Invitrogen. Alginic acid sodium salt from brown algae (Fluka 71238, 50g) and Dextran from leuconostoc ssp. (Fluka 31389, $\mathrm{M}_{\mathrm{r}}=40.000 \mathrm{~g} / \mathrm{mol}, 100 \mathrm{~g}$ ) were from Fluka Biochemika. Sucrose 99\% was from Alfa Aesar (A15583 L 13300, 2.5g) and D-(+)-glucose from Sigma Aldrich (G5767-500g). Solvents from Sigma Aldrich for fluorophore labeling (DMSO and DMF) were anhydrous. All products were used as received unless otherwise specified.

Poly(trimethylene carbonate) $)_{30}-b$-poly(L-glutamic acid $)_{19} \mathrm{PTMC}_{30}-b-\mathrm{PGA}_{19}$ diblock copolymer was synthesized by ring-opening polymerization (ROP) of $\delta$-benzyl-L-glutamate $\mathrm{N}$-carboxyanhydride (NCA) initiated by a primary amine end-functionalized PTMC macroinitiator according to a previously

described method. ${ }^{55}$ All the experiments described were performed on $\mathrm{PTMC}_{30}-b$-PGA 19 diblock copolymer $\left(\mathrm{M}_{\mathrm{n}}=5492 \mathrm{~g} / \mathrm{mol}, \mathrm{I}=2\right)$.

\subsection{Fluorescent dye labeling.}

- Alexa Fluor 568 labeled PTMC $30-b-P A_{19}:$ After flame-drying of a round-bottom flask under vacuum, the following reagents were introduced under inert nitrogen atmosphere: primary end- 


\section{Langmuir}

functionalized amine $\mathrm{PTMC}_{30}-b-\mathrm{PGA}_{19}(99.9 \mathrm{mg}, 18.2 \mu \mathrm{mol})$, anhydrous DMSO (2 mL), DIPEA (140 $\mu \mathrm{L}, 804 \mu \mathrm{mol})$ and reactive Alexa Fluor 568-carboxylic acid, succinimidyl ester (1 mg, $1.26 \mu \mathrm{mol})$ in DMSO $(120 \mu \mathrm{L})$. The reaction was then allowed to proceed for one night under static nitrogen atmosphere. The conjugate $\mathrm{PTMC}_{30}-b$ - $\mathrm{PGA}_{19}$-Alexa Fluor 568 was purified by dialysis $(5 \mathrm{~L}, 3 \mathrm{~h}$, MWCO : $3500 \mathrm{~g} / \mathrm{mol}$ ) after dilution in the flask by DMSO for a better recovery. External medium was renewed six times in course of dialysis. After lyophilization, the conjugate was recovered in a $97 \%$ yield (determined by gravimetry).

- Alexa Fluor 488 labeled poly(butadiene): After flame-drying under vacuum of a round-bottom flask, the following reagents were introduced under inert nitrogen atmosphere: primary end-functionalized amine $\mathrm{PB}_{20}(84.1 \mathrm{mg}, 49.5 \mu \mathrm{mol})$, anhydrous DMF $(2 \mathrm{~mL})$, DIPEA $(8 \mu \mathrm{L}, 45.9 \mu \mathrm{mol})$ and reactive Alexa Fluor 488-carboxylic acid, succinimidyl ester $(1 \mathrm{mg}, 1.55 \mu \mathrm{mol})$ in DMF $(120 \mu \mathrm{L})$. The reaction was then allowed to proceed for overnight under static nitrogen atmosphere. After concentrating the mixture by evaporation of DMF under vacuum, the polymer was precipitated and washed with water. Finally, it was dissolved in THF and recovered after drying under dynamic vacuum.

$\underline{2.3}$. Nanoprecipitation yielding nanosize polymersomes of $\mathrm{PTMC}_{30} \underline{-b-\mathrm{PGA}} \underline{19}$ for encapsulation inside giant polymersomes.

For the preparation of fluorescently labeled nanosize polymersomes to be tracked by confocal microscopy, we followed a nanoprecipitation method described previously. ${ }^{55}$ Briefly, $4.5 \mathrm{~mL}$ Tris Buffer, $50 \mathrm{mM}, \mathrm{pH} 7.4$ was added slowly at a controlled rate of $2.25 \mathrm{~mL} / \mathrm{h}$ on a DMSO solution containing $5 \mathrm{mg}$ of PTMC-b-PGA-Alexa Fluor $568(0.5 \mathrm{~mL})$ under stirring at $500 \mathrm{rpm}$ at $25{ }^{\circ} \mathrm{C}$. The sample was then dialyzed with a 50,000 g/mol cut-off in 5 L Milli Q water with 3 renewals. Size and polydispersity were characterized by dynamic light scattering (DLS).

2.4. Emulsion-centrifugation yielding giant PB- $b$-PEO encapsulating a PTMC- $b$-PGA nanosize vesicle 
suspension (Scheme 1).

In a typical procedure inspired from $\mathrm{Li}$ and col., ${ }^{56,57} 5 \mu \mathrm{L}$ of a nanosize vesicle suspension in 380 mOsm sucrose solution was first poured in $500 \mu \mathrm{L}$ toluene containing $3 \mathrm{mg} / \mathrm{mL}$ PB- $b$-PEO (including, depending on the experiment, 0 or $10 \mathrm{wt} \%$ of Alexa Fluor 488 labeled PB) in a Eppendorf tube (step 1, Scheme 1). The PB-b-PEO solution in toluene was previously stirred for at least 2 hours to ensure a complete dissolution of the copolymer, as verified by DLS (no intensity scattered). In another tube (step 2, Scheme 1), $30 \mu \mathrm{L}$ of the same organic solution was poured over $30 \mu \mathrm{L}$ of a $380 \mathrm{mOsm}$ aqueous glucose solution and allowed to stabilize for $30 \mathrm{~min}$. Finally (step 3, Scheme 1), the first tube was emulsified with vigorous agitation by hand yielding quite homogeneous (see Figure 1) inverted emulsion droplets (alternatively, repeated pipetting works as well). Then $50 \mu \mathrm{L}$ of this emulsion was poured slowly over the second interface tube. The sample was then immediately centrifuged at $20{ }^{\circ} \mathrm{C}$ at $500 \mathrm{~g}$ for $4 \mathrm{~min}$ and the aqueous polymer vesosome suspension was recovered in the lower phase (Figure 2). 




\section{2. interface $w / o$}

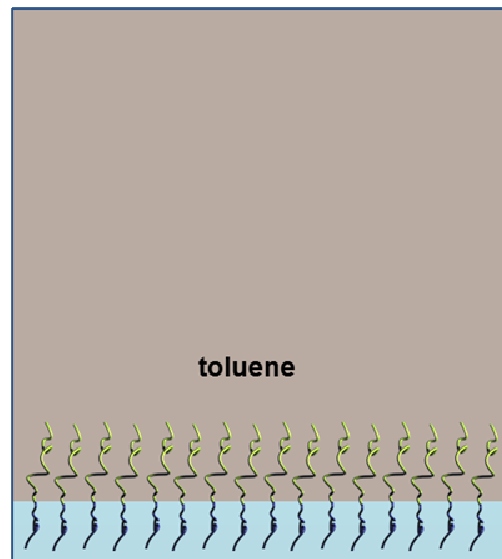

glucose $\mathbf{3 8 0}$ mOsm in water

\section{3. centrifugation}

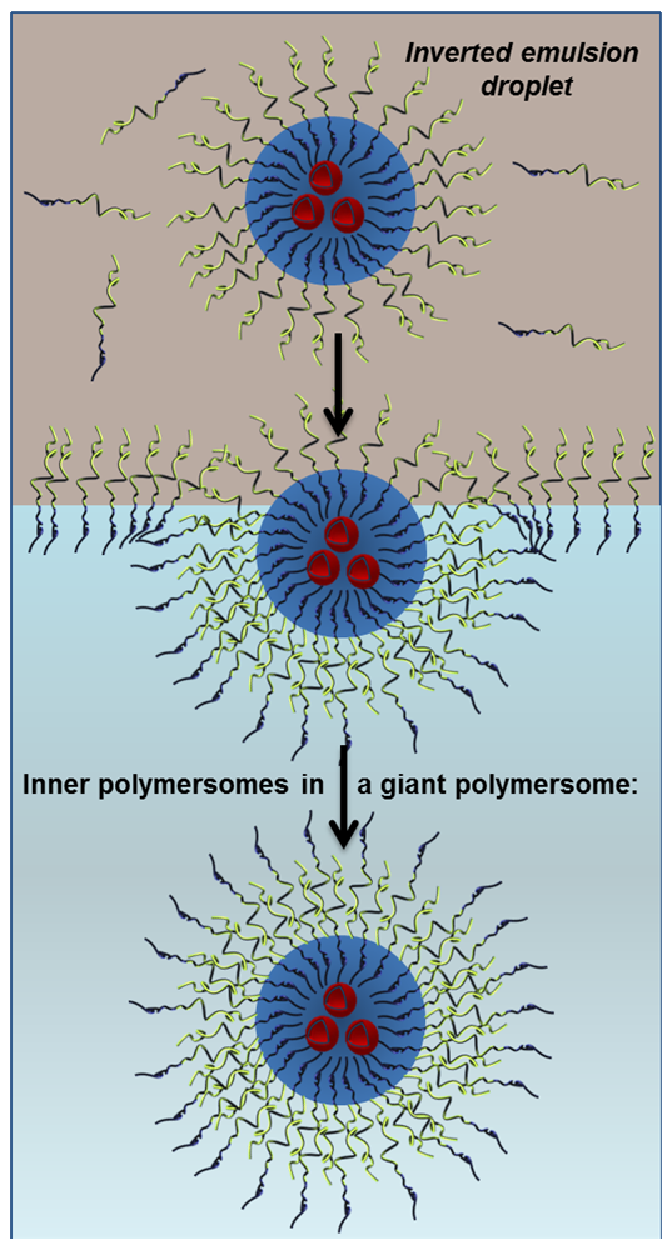

Scheme 1. Scheme of the emulsion-centrifugation process yielding either giant polymersomes (Figure 2) or polymersomes in polymersomes (when, like here a suspension of nanosize polymersomes (in red) is used as inner aqueous phase of the w/o emulsion).



Figure 1. Optical microscopy acquisitions of the w/o emulsion stabilized by poly(butadiene)- $b$ poly(ethylene oxide). From left to right: bright field, epifluorescence (green channel), epifluorescence (red channel) and overlay of red and green channels. The green channel features the encapsulated FITC- 


\section{Langmuir}

dextran $(1 \mathrm{mg} / \mathrm{mL})$, the red channel the Nile Red $(0.05 \mathrm{mg} / \mathrm{mL})$ solubilized in toluene.

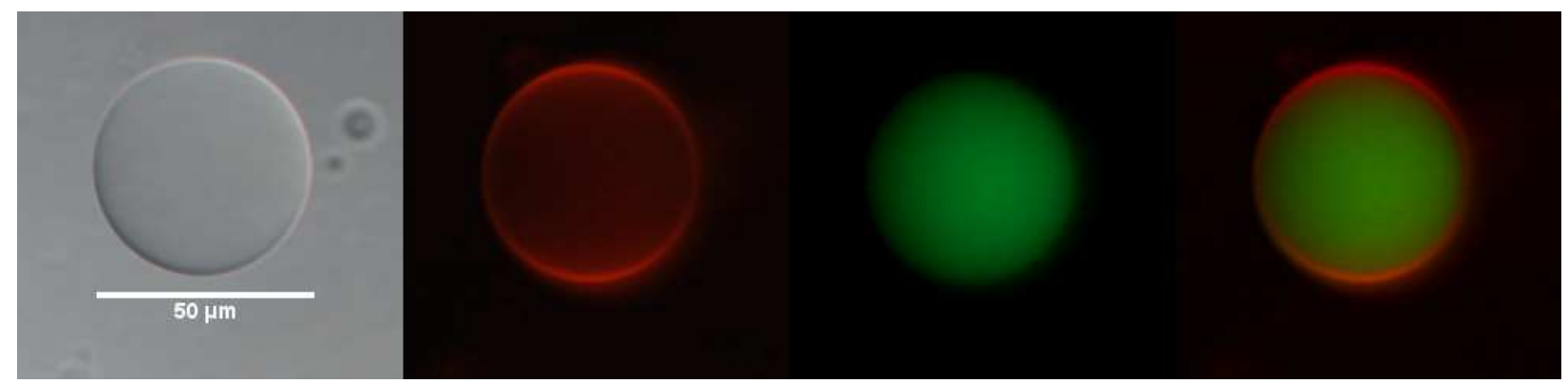

Figure 2. Microscopy acquisitions of a giant polymersome of poly(butadiene)- $b$-poly(ethylene oxide). From left to right, bright field microscopy, red channel epifluorescence with Nile Red membrane labeling $(0.05 \mathrm{mg} / \mathrm{mL})$, green channel with $10.000 \mathrm{~g} / \mathrm{mol}$ FITC-dextran $(1 \mathrm{mg} / \mathrm{mL})$, and overlay of red and green.

The process has been described to quantitatively encapsulate hydrophilic solutions, ${ }^{58}$ such as FITCdextran as shown in Figure 2. Of course, any aqueous solution can be encapsulated using this method. For experiments regarding "cytoplasm mimic", the appropriate amount of respectively alginate/dextran was added to the nanosize polymersome suspension (at $380 \mathrm{mOsm}$ sucrose) to a final concentration of respectively $10 / 300 \mathrm{mg} / \mathrm{mL}$. In both cases, the centrifugation time was reduced to $3 \mathrm{~min}$.

\section{$\underline{\text { 2.5. Methods }}$}

Dynamic Light Scattering (DLS). DLS measurements were conducted on a Malvern Zeta Sizer Nano ZS instrument with $90^{\circ}$ angle analysis. The mean hydrodynamic diameter and its distribution were determined using Cumulant and CONTIN methods. For particle tracking, viscosity and refractive index were determined for each solution using a rheometer (AR2000) and an Abbe refractometer.

Spinning disk confocal microscopy. The spinning disk microscope was a Leica DMI6000 (Leica Microsystems, Wetzlar, Germany) equipped with a confocal Scanner Unit CSU-X1 (Yokogawa) using for this experiment objective HCX PL Apo 100X oil NA 1.4 and an Evolve EMCCD camera (Roper 


\section{Langmuir}

Scientific, Evry, France). Z-stack analysis was performed with a galvanometric stage (Leica Microsystems, Wetzlar, Germany). The diode laser excitation wavelengths used were $491 \mathrm{~nm}$ and 561 $\mathrm{nm}$ and a Semrock emission filter with narrow bandpass windows in the blue (420 to $460 \mathrm{~nm}$ ), green (506 to $536 \mathrm{~nm})$ red (587 to $627 \mathrm{~nm})$ and near infrared (670 to $730 \mathrm{~nm})$ spectral regions was used. Microscopy chambers were fabricated by sealing a slide against a coverslip with two layers of Parafilm $^{\mathrm{TM}}$ featuring the three sides of the chamber. The sample was then injected by capillarity through the last open side. Finally, the last aperture was sealed with molten paraffin wax. Experiments were carried out in the Bordeaux Imaging Center of the University of Bordeaux Segalen. The help of Sébastien Marais is gratefully acknowledged, particularly for 3D reconstruction of z-stacks with the Imaris software.

Epifluorescence and optical microscopy. Bright field and fluorescence microscopy images of giant polymersomes (Figure 1 and 2) were taken on a Zeiss Axiovert 40 CFL inverted microscope with a EC Plan-NEOFLUAR 40x DIC Na 0.75 objective captured with a 2Mbytes digital Gigabit Ethernet CCD camera (Viewvorks VG-2M, South Korea). For epifluorescence microscopy, a mercury lamp was used as source with excitation and emission filters of narrow bandpass windows in the green (464.5 to 499.5 $\mathrm{nm}$ for excitation and 516 to $556 \mathrm{~nm}$ for emission) and red (532 to $544 \mathrm{~nm}$ for excitation and 573 to 637 $\mathrm{nm}$ for emission) spectral regions (provided by Semrock). The samples were placed between a glass slide and a coverslip separated by a Parafilm ${ }^{\mathrm{TM}}$ spacer as mentioned above for confocal microscopy imaging.

Particle tracking The 2D Brownian motion of inner polymersomes labeled in red and encapsulated in a giant polymersome (Movie S1 in ESI) was tracked down. The 2D position $\left(\mathrm{x}_{\mathrm{M}}, \mathrm{y}_{\mathrm{M}}\right)$ for each frame and each nanosize vesicle of the $50 \mathrm{~s}$ movie (500 frames and $\tau=100 \mathrm{~ms}$ ) was reported. By linking the trajectories (79 in total) for each nanosize vesicle frame by frame, we have access to displacements $\Delta \mathrm{x}$, $\Delta y$ (788 in total) versus time. For the polymer vesosome with the "cytoplasm mimick" Dextran, 187 frames of a longer movie (Movie S2 in ESI) were tracked yielding 810 displacements (56 trajectories). 


\section{Langmuir}

\subsection{Instrumentation and Measurements.}

${ }^{1} \mathrm{H}(400 \mathrm{MHz})$ Nuclear Magnetic Resonance NMR spectra were recorded on a Bruker Avance DPX400 instrument at $23{ }^{\circ} \mathrm{C}$ and were referenced internally using the residual ${ }^{1} \mathrm{H}$ solvent resonance relative to tetramethylsilane $(\delta=0) . M_{n}$ SEC and $\left(M_{w} / M_{n}\right)$ values for copolymers PTMC- $b$-PBLG (3 mg/mL) were determined by Size Exclusion Chromatography SEC at $60{ }^{\circ} \mathrm{C}$, using dimethylformamide (DMF) with $\operatorname{LiBr}(1 \mathrm{~g} / \mathrm{L})$ as eluent $(0.8 \mathrm{~mL} / \mathrm{min})$, on a Jasco apparatus equipped with both Varian refractive index and UV detectors and two PL gel $5 \mu \mathrm{m}$ mixed-C columns.

\section{Results and Discussion}

\section{$\underline{\text { 3.1. Formation of biomimetic compartmentalized polymersomes. }}$}

Thanks to the emulsion-centrifugation process ${ }^{56,57}$ and its quantitative loading efficiency ${ }^{58}$ (see Scheme 1, Experimental section), a suspension of red fluorescent Alexa 568 labeled polymersomes (or nanosize vesicles) was encapsulated in a green fluorescent Alexa 488 labeled giant polymersome, as shown in Figure 3 where inner red PTMC- $b$-PGA polymersomes can clearly be seen in a green giant PB- $b$-PEO polymersome. Movie S1 in ESI shows the Brownian motion of these inner nanosize polymersomes. The $3 \mathrm{D}$ reconstruction of the $\mathrm{z}$-stack observation by spinning disk confocal microscopy of this polymer vesosome, evidences the localization of each nanosize polymersome inside the internal volume of the larger vesicle (Figure 3, Movie S2 ESI).



Figure 3. Spinning disk confocal microscopy acquisitions of a polymer vesosome. From left to right, green channel (membrane of the giant polymersome), red channel (nanosize inner polymersomes), 
overlay, and 3D reconstruction of $\mathrm{z}$ stack in red channel.

Such compartmentalized structures can be named polymer vesosomes ${ }^{18,19,22,59}$, polymersomes in polymersomes or double polymersomes ${ }^{21,60}$. Only three groups ${ }^{21,30,31}$ have tackled the challenge of compartmentalized polymersomes so far. We present here an original, facile, versatile, reproducible and low-product consuming technique. Only the microfluidic method recently developed by Weitz and coworkers $^{21}$ presents in our opinion a better process control. However, the internal polymersome size is limited in that case to micrometric one by the process itself and the capillary diameters. The obtained morphology roughly resembles to organelles in a cell which are lipid bilayer compartments (endosomes, lysosomes, mitochondria...) encapsulated themselves in the plasmic membrane.

One step further to challenge cellular biomimicry consists in adding another component with a relevant structure and function, the cytoplasm. In addition to the obvious mechanical properties given by the cytoskeleton, the high macromolecular concentrations found intracellularly are also responsible for the so-called macromolecular crowding effect. ${ }^{45,}{ }^{51,61}$ In 2001, R. John Ellis and col. ${ }^{61}$ launched a call to biochemists to stop neglecting this "macromolecular crowding" in their studies, which is known in polymer science as the "excluded volume effect". All together, macromolecules in the cytoplasm, cytoskeleton and internal compartments occupy 20-30 vol.\% of a cell, generating a strong steric repulsion between them. The consequences on the cell machinery have been rarely considered: for instance, most biochemical reactions are studies in dilute (ideal) solutions, while in real cells one should consider the activity coefficients for both thermodynamic and kinetic studies. He thus advises to use crowding agents under the following criterions: a molecular weight ranging from 50,000 to 200,000 $\mathrm{g} / \mathrm{mol}$, a high water solubility, not being prone to self-aggregation, and last but not least, no interaction with the tested system other than steric repulsion.

In a first attempt, the nanosize polymersome suspension was thus mixed with a highly water-soluble 


\section{Langmuir}

alginate at a final concentration of $10 \mathrm{mg} / \mathrm{mL}$ (Figure 4). For this strongly charged natural polymer, the molar mass distribution was reported in the literature: $M_{\mathrm{n}}=107700 \mathrm{gmol}^{-1}$ and $M_{\mathrm{w}}=231500 \mathrm{gmol}^{-1}{ }^{62}$ so $^{2}$ that the molar concentration of Alginate is $0.1 \mathrm{mM}$ in our case. A $2 \mathrm{wt} . \%$ solution of this product has a viscosity of $1.07 \mathrm{~Pa} . \mathrm{s},{ }^{63}$ therefore the viscosity is around $0.5 \mathrm{~Pa} . \mathrm{s}$ at $10 \mathrm{mg} / \mathrm{mL}$. Thus we can predict a 350-fold reduction of the diffusion coefficient compared to the pure sucrose solution (0.00144 Pa.s). Actually, as observed in Movie S3 (ESI), the motion of inner nanosize polymersomes is not only hampered but in fact completely blocked. One can assume that the high alginate concentration provokes a phase separation phenomenon induced by depletion layers around the PTMC- $b$-PGA vesicles excluding the alginate chains (Figure 4). The diffusion of spherical particles inside a concentrated macromolecular solution with a depletion layer of the chains around the spheres has been considered in theory, predicting anomalous diffusion with mean square displacements not linear versus time. ${ }^{64}$ The case of our giant vesicles containing nanosize vesicles mixed with rather stiff alginate polyelectrolyte chains is even more dreadful since the diffusion is totally arrested. The fact that both alginate and the PGA chains (present on the outer shell of the nanosize polymersomes) are negatively charged must also play an important role in this segregation process, due to additional electrostatic repulsion forces. The 3D reconstruction of this vesosome (Figure 4 and Movie S4 in ESI) clearly evidences different clusters of inner PTMC- $b$-PGA polymersomes in the volume that always seem pushed against the "plasmic membrane" mimic; this is presumably due to the alginate chains exerting an osmotic pressure $\Pi$ on them estimated around $260 \mathrm{~Pa}$ from the molar concentration of chains. The potential between two vesicles brought at contact by depletion attraction can be estimated by $2 \pi \cdot \Pi \cdot R_{v} \cdot R_{a}{ }^{2} \approx 220 k_{B} T$, ${ }^{65}$ which explains why it overcomes thermal motion. 


\section{Langmuir}

\section{$10 \mu \mathrm{m}$}

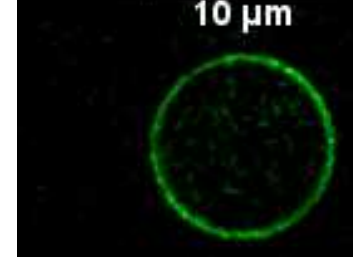

Figure 4. Spinning disk confocal microscopy acquisitions of a polymer vesosome with "cytoplasm mimic" alginate in the cavity of the giant polymersome. From left to right, green channel (membrane of the giant polymersome), red channel (nanosize inner polymersomes), overlay and 3D reconstruction in red channel.

In another set of experiments, a $40.000 \mathrm{~g} / \mathrm{mol}$ neutral dextran was used as cytoplasm mimic at a concentration of $300 \mathrm{mg} / \mathrm{mL}$. This concentration roughly corresponds to an intracellular macromolecular concentration of 20-30 vol.\% and can be considered realistic to mimic the intracellular crowding. ${ }^{61}$ For example, the total concentration of protein and RNA inside a cell of Escherichia coli lies between $300-400 \mathrm{mg} / \mathrm{mL}$, in good correlation with our simplified synthetic model. ${ }^{66}$ Finally, the choice of dextran is also motivated by the fact that its use is accepted in hard particle excluded volume models at high concentration for crowding effect. ${ }^{61}$ The resulting polymer vesosomes obtained in these conditions are reported in Figure 5. As observed on Movie S5 (ESI), motion of the inner polymersomes is now considerably decreased and hindered, but not blocked, clearly illustrating this crowding effect at high volume fractions of macromolecules in a confined volume.

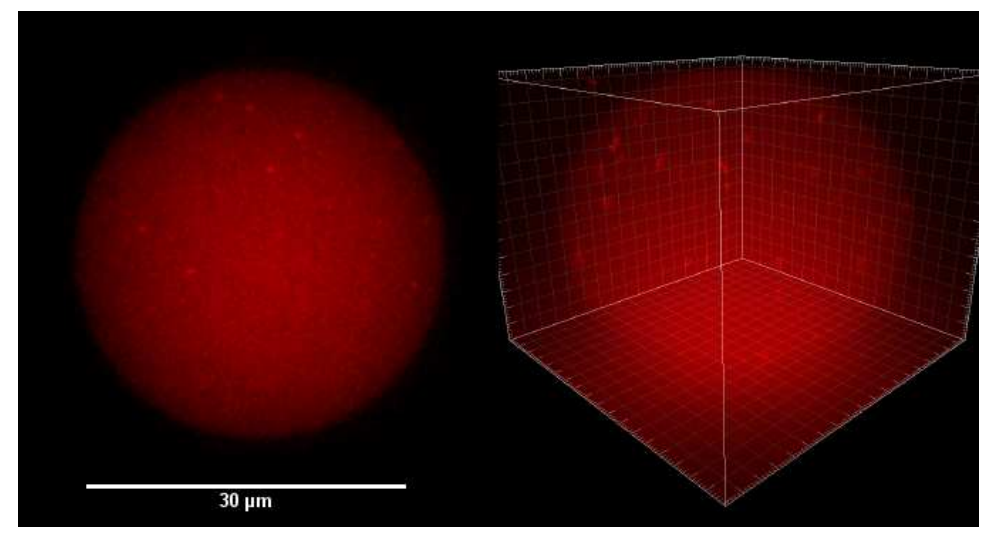

Figure 5. Spinning disk confocal microscopy acquisitions of a polymer vesosome with "cytoplasm mimic" dextran in the cavity of the giant polymersome. Red channel (nanosize inner polymersomes), 


\section{Langmuir}

and $3 \mathrm{D}$ reconstruction in red channel.

\subsection{Quantitative analysis of the dynamics of internal vesicles}

The observation of the stochastic motion of the nanosize polymersomes alone seems to be in agreement with a Brownian motion, showing that their loading into a giant polymersome does not affect their dynamic properties. In order to quantitatively analyze this parameter, the $2 \mathrm{D}$ positions of each distinct nanosize vesicle in Movie S1 were tracked $(\tau=100 \mathrm{~ms})$ and their displacement from frame to frame calculated. $788 \Delta \mathrm{x}$ and $\Delta \mathrm{y}$ displacements or in other words 79 full trajectories could be obtained. The frequency of these displacements versus $\Delta \mathrm{x}$ and $\Delta \mathrm{y}$ is plotted in Figure 6.

In statistical physics, the normal (or Gaussian) distribution (Equation 1) is usually associated to random walks like particles in Brownian motion. In the case of pure diffusion (no translation), the mean position $\langle x\rangle=\mu$ is zero, while the mean square displacement $\left\langle x^{2}\right\rangle$ is linear in time with a slope equal to $2 \sigma^{2}$ (in one dimension).

$$
f(x)=\frac{1}{\sqrt{2 \pi \sigma^{2}}} \cdot e^{\frac{(x-\mu)^{2}}{2 \sigma^{2}}}
$$

Equation 1

The presented data can be well fitted with a Gaussian distribution (Figure 6a), attesting that the motion of nanosize polymersomes is not affected by their encapsulation in a polymersome of an approximately $20 \mu \mathrm{m}$ diameter. However, the outer membrane could have restricted the diffusion of the small vesicles in some limiting volume (as a cage). This is not the case because the diameter of the giant vesicle is much larger $(20 \mu \mathrm{m})$ than the mean square displacement of the small internal ones and is also prone to a slight translational diffusion itself. Another way to analyze the data consists in representing the mean square displacements of some of the longest lasting trajectories in a log-log representation (Figure 6b). 


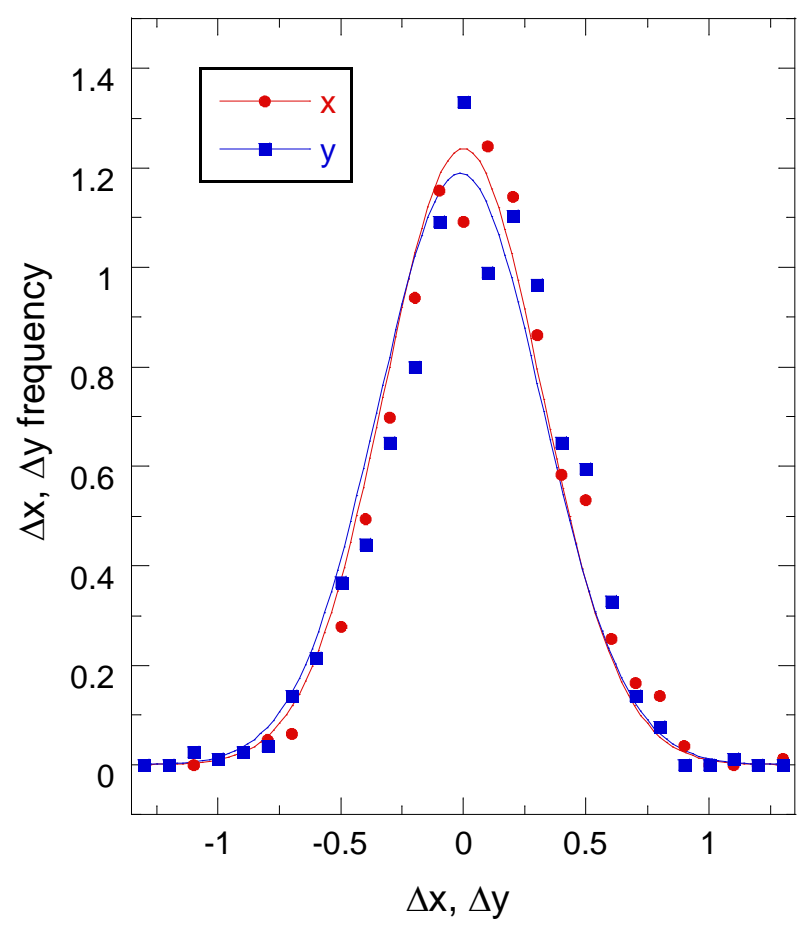

(a)

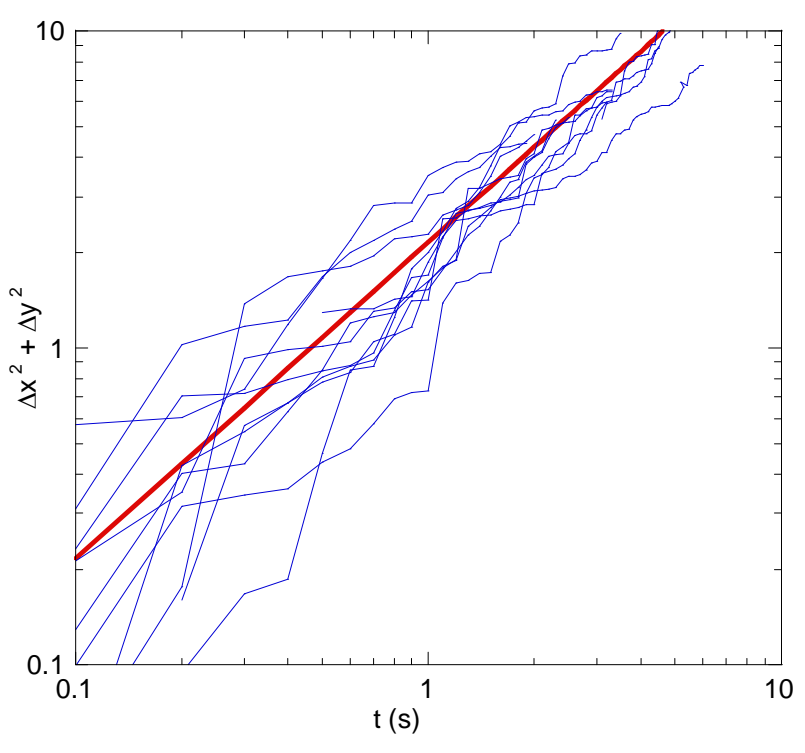

(b)

Figure 6. (a) Statistics of displacements $(\mu \mathrm{m})$ in $\mathrm{x}$ (red circles) and y (blue squares) directions of nanosize inner polymersomes in vesosome corresponding to Figure 3 (Movie S1 ESI). (b) Mean square displacement $\Delta \mathrm{x}^{2}$ and $\Delta \mathrm{y}^{2}\left(\mu \mathrm{m}^{2}\right)$ plotted versus time (s). Blue lines represent the experimental trajectories. The red line features the model trajectory with the mean calculated diffusion coefficient.

The trajectories represented here have the same slope in log-log representation than a model trajectory $\stackrel{2}{r}=\Delta x^{2}+\Delta y^{2}=4 D_{\text {diff }} \times t \quad$ estimated with the mean Diffusion coefficient (see Equation 2) calculated for these nanosize vesicles, again in agreement with purely diffusive (Brownian) motion. The scattering of the prefactor $\left(4 D_{\text {diff }}\right)$ for the shown trajectories in blue is ascribed to the inherent size-dispersity of the internal vesicles (see Table 1).

With the $788 \Delta x$ and $\Delta y$ elementary steps, an average diffusion coefficient $D_{D i f f}$ can then be estimated following equation 2, and be converted into a hydrodynamic diameter $R_{H}$ using the well-known Stokes Einstein relation (Equation 3).

$$
D_{\text {diff }}\left(\frac{\mu m^{2}}{s}\right)=\frac{\sigma^{2}\left(\mu m^{2}\right)}{2 \tau(s)}
$$

Equation 2 


$$
R_{H}=\frac{k_{B} \cdot T}{6 \cdot \pi \cdot D_{\text {diff }} \cdot \eta}
$$

Equation 3

The obtained $D_{\text {Diff }}$ and $R_{H}$ values can now be quantitatively compared to data resulting from the nanosize polymersomes initially prepared (Table 1). The values measured in solution by DLS are in excellent correlation with the ones determined inside giant polymersomes by video tracking, attesting the validity of the method and confirming quantitatively the randomness of the vesicles motion.

Table 1. Characteristics of nanosize polymersome suspension before and after encapsulation in a giant polymersome

\begin{tabular}{|c|c|c|c|c|c|}
\hline \multicolumn{5}{|c|}{$\begin{array}{c}\text { Without cytoplasm mimic } \\
\text { (380 mM sucrose, } \eta=0.00144 \text { Pa.s }\end{array}$} & \multirow{2}{*}{$\begin{array}{c}\text { With cytoplasm mimic } \\
\text { (dextran } 300 \mathrm{mg} / \mathrm{mL}, 380 \\
\mathrm{mM} \text { sucrose, } \\
\left.\eta=0.0367 \mathrm{~Pa} . \mathrm{s}^{\mathrm{a}, \mathrm{e}}\right) \\
D_{\text {diff }^{\mathrm{c}}\left(\mu \mathrm{m}^{2} / \mathrm{s}\right)}\end{array}$} \\
\hline$\underset{(\mathrm{nm})}{\operatorname{Mean} R_{\mathrm{H}}}$ & $\mathrm{PDI}^{\mathrm{b}}$ & $\begin{array}{l}\text { Mean } D_{\text {diff }}{ }^{b} \\
\left(\mu \mathrm{m}^{2} / \mathrm{s}\right)\end{array}$ & $\underset{(\mathrm{nm})}{\operatorname{Mean} R_{\mathrm{H}}^{\mathrm{c}}}$ & $\underset{(\mu \mathrm{m} / \mathrm{s})}{\operatorname{Mean} D_{\text {diff }}^{\mathrm{c}}}$ & \\
\hline $\begin{array}{c}257 \pm 9 \\
(\text { Contin })^{d}\end{array}$ & $0.1 \pm 0.02$ & $0.718 \pm 0.004^{d}$ & $271 \pm 21^{e}$ & $0.55 \pm 0.042^{e}$ & $0.082 \pm 0.011^{d}$ \\
\hline
\end{tabular}

For each value, error represents the standard error $\sigma / \sqrt{n}$ where $n$ represents the numbers of trajectories or measurements ${ }^{a}$ Determined by rheometry ${ }^{b}$ Determined by Dynamic Light Scattering in $380 \mathrm{mM}$ sucrose. PDI means Polydispersity Index. ${ }^{c}$ Determined by particle tracking in $380 \mathrm{mM}$ sucrose with and without dextran $300 \mathrm{mg} / \mathrm{mL}$. ${ }^{d}$ determined at $21^{\circ} \mathrm{C}$. ${ }^{e}$ determined at $25^{\circ} \mathrm{C}$.

It seems visible in Movie S5, that the presence of $300 \mathrm{mg} / \mathrm{mL}$ dextran considerably and efficiently hinders the motion of the nanosize vesicles. Once again, their 2D positions was tracked from Movie S5, yielding to $810 \Delta \mathrm{x}$ and $\Delta \mathrm{y}$ displacements or in other words 56 full trajectories. The frequency of the displacements versus $\Delta \mathrm{x}$ and $\Delta \mathrm{y}$ is plotted in Figure $7 \mathrm{a}$ and data properly fitted with a Gaussian distribution. 


\section{Langmuir}

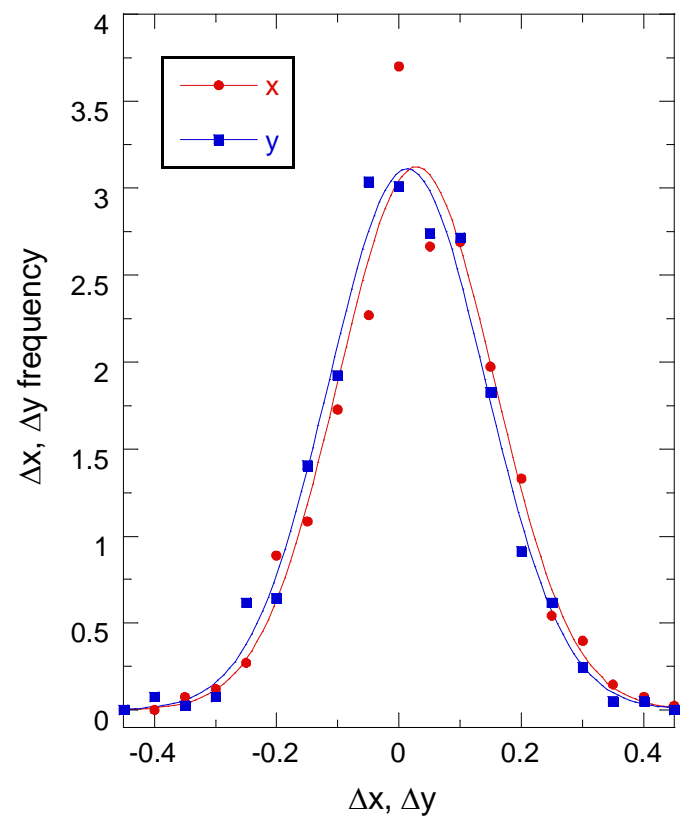

(a)

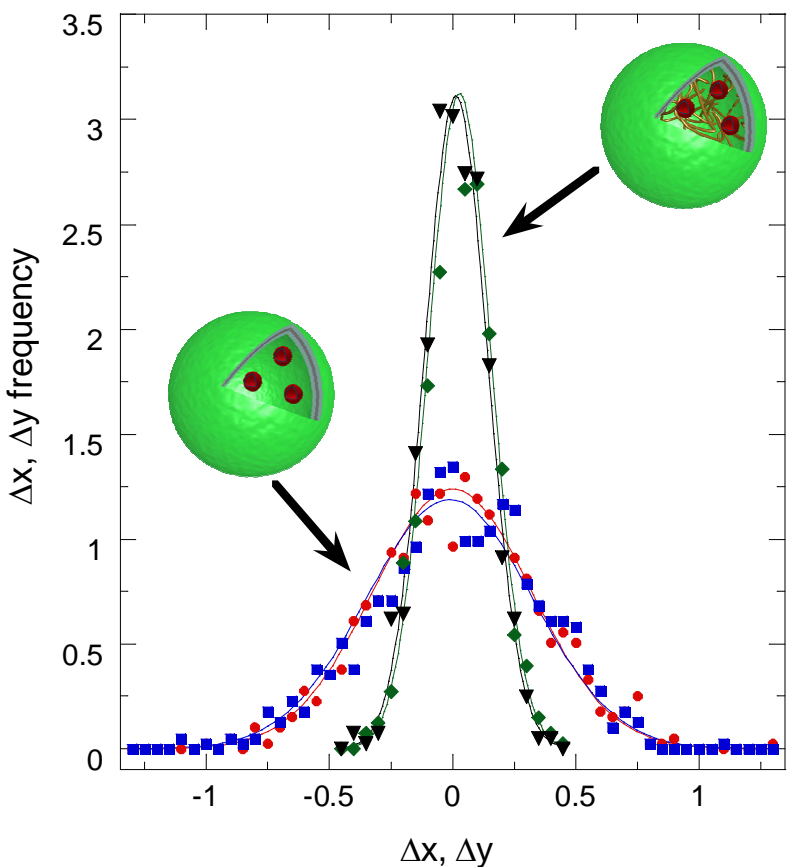

(b)

Figure 7. (a) Displacement $\Delta x$ (red circles) and $\Delta y$ (blue squares) frequency of nanosize inner polymersomes in $300 \mathrm{mg} / \mathrm{mL}$ dextran in giant polymersome, corresponding to Figure 5 and Movie S5 (ESI). (b) Comparison of displacement frequency without and with Dextran: overlay of Figures 6a and $7 \mathrm{a}$.

The value of the diffusion coefficient of the nanosize polymersomes calculated in these "cytoplasm mimic" conditions is nearly 6.6 times slower than in low viscosity conditions (Table 1, Figure 7b). This significant decrease is however lower than the approximately 30-fold increase of viscosity for this solution of dextran compared to the initial aqueous solution. For this dextran $\mathrm{T} 40\left(\mathrm{M}_{\mathrm{r}}=40000 \mathrm{gmol}^{-1}\right)$, M. Prouty and R. Podgornik derived a power law of osmotic pressure versus weight concentration, ${ }^{67,68}$ from which we calculate $5.3 \times 10^{5} \mathrm{~Pa}$ for a $300 \mathrm{mg} / \mathrm{mL}$ concentration. Prepared initially in pure water, the nanosize PTMC- $b$-PGA vesicles are submitted to hypertonic conditions inside the giant PB- $b$-PEO vesicle: the osmotic pressure exerted both by dextran and by $380 \mathrm{mM}$ sucrose is estimated around $1.5 \times 10^{6} \mathrm{~Pa}$. Therefore the hydrodynamic size of the PTMC- $b$-PGA vesicles might decrease by osmotic deflation through water permeability across their membrane. Another explanation going in the same direction is the increase of the Brownian diffusion constant of vesicles due to the crowding effect (the 


\section{Langmuir}

volume taken by dextran chains being excluded to them). S. Longeville and coworkers have indeed evidenced by SANS a two-fold compaction of coil chains such as PEG in a 30 vol.\% solution of a crowding agent such as the Ficoll 70 polysaccharide, exerting an osmotic pressure around $3 \times 10^{5} \mathrm{~Pa}^{69,70}$ Therefore we can suspect a similar effect on the hydrophilic blocks of the polymersomes' membranes (PGA for the small vesicles, PEG for the giant vesicles). The two effects combined (osmotic deflation and hydrophilic chains compaction by the crowding agent) are explaining why particle tracking yielded a 6.6-fold decrease of the diffusion constant of the internal vesicles, while the ratio of viscosities with and without dextran is larger (about 30-fold). Finally, the viscosity of the dextran system (Table 1) is of the order of magnitude of the usual cytoplasm viscosity reported for red blood cells (0.01 Pa.s), ${ }^{35}$ thereby confirming that is was a good choice for mimicking the interior of cells on a physical point of view.

\section{Conclusion}

In this work, we demonstrated the formation of artificial structural cell mimics with organelles and a "model cytoplasm" with a facile, versatile, reproducible and low product and time-consuming technique. To the best of our knowledge, this is the first report where both these aspects have been combined, especially with polymersomes. A suspension of nanosize inner polymersomes of PTMC- $b$ PGA (formed by nanoprecipitation) is encapsulated in giant polymersomes of PB- $b$-POE together with highly viscous alginate or dextran solutions, thanks to the emulsion-centrifugation process. The formation of this biomimetic structure was evidenced by fluorescence confocal microscopy. Moreover, the $2 \mathrm{D}$ motion of these artificial organelles was tracked down and confirmed as still being Brownian inside the volume of an approximately $20 \mu \mathrm{m}$ giant polymersome. This analysis was repeated in presence of "cytoplasm mimic dextran", their motion being efficiency hindered as confirmed by a 6.6 times smaller diffusion coefficient. Furthermore, the concentration of $300 \mathrm{mg} / \mathrm{mL}$ of polysaccharide (dextran T40) brings a viscosity above 0.01 Pa.s, in the range of red blood cell cytoplasm viscosity, a 
volume fraction near $30 \%$ and an osmotic pressure above $1 \mathrm{MPa}$ resembling the intra-cellular conditions caused by global cellular proteins. By reproducing the intracellular "macromolecular crowding effect" which plays a crucial role in the cell machinery, we believe that this synthetic and simplified approach constitutes an appropriate cytoplasm mimic. Even with such simplified cell mimics, soft materials with innovative properties can arise and extend the domain of possibilities in the fields of cosmetics, fragrance encapsulation, drug delivery and fine chemical additives.

\section{ACKNOWLEDGMENT}

This work was financially supported by the French Ministère de l'Enseignement Supérieur et de la Recherche. The confocal microscopy acquistions were done in the Bordeaux Imaging Center of the University Bordeaux Segalen. The help of Sébastien Marais is acknowledged, particularly for 3D reconstruction. The authors are grateful to Dr. Julie Thévenot for PTMC- $b$-PGA synthesis and to Laurent Bui for work art contributions. P2M program from the European Science Foundation is also gratefully acknowledged.

\section{Supporting Information Available}

Movie S1 acquired with a spinning disk confocal microscopy featuring the polymer vesosome in Figure 3, where the loaded red inner polymersomes can be clearly observed in Brownian motion as they are labeled with Alexa Fluor 568. Movie S2 with the vesosome of Movie S1 reconstructed in three dimensions showing the localization of the inner polymersomes in the giant one. Movie S3 featuring inner red-labeled polymersomes blocked in alginate loaded in the giant polymersome of Figure 5. Movie S4 featuring the 3D reconstruction of the vesosome with alginate ("cytoplasm mimick") in Movie S3. Movie S5 featuring inner red-labeled polymersomes in hindered motion in dextran (“cytoplasm mimick") loaded in a giant polymersome. Movie S6 featuring the 3D reconstruction of the 
vesosome with Dextran in Movie S5. 


\section{REFERENCES}

(1) Discher, B. M.; Won, Y. Y.; Ege, D. S.; Lee, J. C. M.; Bates, F. S.; Discher, D. E.; Hammer, D. A., Science 1999, 284 (5417), 1143-1146.

(2) Discher, D. E.; Eisenberg, A., Science 2002, 297 (5583), 967-973.

(3) Bermudez, H.; Brannan, A. K.; Hammer, D. A.; Bates, F. S.; Discher, D. E., Macromolecules 2002, 35 (21), 8203-8208.

(4) Brinkhuis, R. P.; Rutjes, F. P. J. T.; van Hest, J. C. M., Polym. Chem. 2011.

(5) Christian, D. A.; Cai, S.; Bowen, D. M.; Kim, Y.; Pajerowski, J. D.; Discher, D. E., Eur. J. Pharm. Biopharm. 2009, 71 (3), 463-474.

(6) Meng, F.; Zhong, Z.; Feijen, J., Biomacromolecules 2009, 10 (2), 197-209.

(7) Cheng, R.; Feng, F.; Meng, F.; Deng, C.; Feijen, J.; Zhong, Z., J. Controlled Release 2011, 152 (1), 2-12.

(8) Li, M.-H.; Keller, P., Soft Matter 2009, 5 (5), 927-937.

(9) Van Dongen, S. F. M.; Verdurmen, W. P. R.; Peters, R. J. R. W.; Nolte, R. J. M.; Brock, R.; Van Hest, J. C. M., Angew. Chem. Int. Ed. 2010, 49 (40), 7213-7216.

(10) Van Dongen, S. F. M.; Nallani, M.; Cornelissen, J. J. L. M.; Nolte, R. J. M.; Van Hest, J. C. M., Chem.--Eur. J. 2009, 15 (5), 1107-1114.

(11) Renggli, K.; Baumann, P.; Langowska, K.; Onaca, O.; Bruns, N.; Meier, W., Adv. Funct. Mater. 2011, 21 (7), 1241-1259.

(12) Onaca, O.; Hughes, D. W.; Balasubramanian, V.; Grzelakowski, M.; Meier, W.; Palivan, C. G., Macromol. Biosci. 2010, 10 (5), 531-538.

(13) Tanner, P.; Baumann, P.; Enea, R.; Onaca, O.; Palivan, C.; Meier, W., Acc. Chem. Res. 2011, dx.doi.org/10.1021/ar200036k.

(14) Schatz, C.; Louguet, S.; Meins, J. F. L.; Lecommandoux, S., Angew. Chem. Int. Ed. 2009, 48 (14), 2572-2575.

(15) Brizard, A. M.; Van Esch, J. H., Soft Matter 2009, 5 (7), 1320-1327.

(16) Hawking, S., The Universe in a Nutshell. Bantam Books: New York, 2001.

(17) Al-Jamal, W. T.; Kostarelos, K., Int. J. Pharm. 2007, 331 (2), 182-185.

(18) Scholl, I.; Boltz-Nitulescu, G.; Jensen-Jarolim, E., J. Controlled Release 2005, 104 (1), 1-27.

(19) Walker, S. A.; Kennedy, M. T.; Zasadzinski, J. A., Nature 1997, 387 (6628), 61-64.

(20) Shum, H. C.; Zhao, Y. J.; Kim, S. H.; Weitz, D. A., Angew. Chem. Int. Ed. 2011, 50 (7), 16481651.

(21) Kim, S.-H.; Shum, H. C.; Kim, J. W.; Cho, J.-C.; Weitz, D. A., J. Am. Chem. Soc. 2011, dx.doi.org/ 10.1021/ja205687k.

(22) Wong, B.; Boyer, C.; Steinbeck, C.; Peters, D.; Schmidt, J.; van Zanten, R.; Chmelka, B.; Zasadzinski, J. A., Adv. Mater. 2011, 23 (20), 2320-2325.

(23) McPhail, D.; Tetley, L.; Dufes, C.; Uchegbu, I. F., Int. J. Pharm. 2000, 200 (1), 73-86.

(24) Bolinger, P. Y.; Stamou, D.; Vogel, H., Angew. Chem. Int. Ed 2008, 47 (30), 5544-5549.

(25) Kreft, O.; Prevot, M.; Möhwald, H.; Sukhorukov, G. B., Angew. Chem. Int. Ed. 2007, 46 (29), 5605-5608.

(26) Kreft, O.; Skirtach, A. G.; Sukhorukov, G. B.; Möhwald, H., Adv. Mater. 2007, 19 (20), 31423145 .

(27) Stadler, B.; Price, A. D.; Chandrawati, R.; Hosta-Rigau, L.; Zelikin, A. N.; Caruso, F., Nanoscale 2009, 1 (1), 68-73.

(28) Chandrawati, R.; Hosta-Rigau, L.; Vanderstraaten, D.; Lokuliyana, S. A.; Stadler, B.; Albericio, F.; Caruso, F., ACS nano 2010, 4 (3), 1351-1361.

(29) Hosta-Rigau, L.; Chung, S. F.; Postma, A.; Chandrawati, R.; Städler, B.; Caruso, F., Adv. Mater. 2011, 23 (35), 4082-4087. 
(30) Fu, Z.; Ochsner, M. A.; De Hoog, H. P. M.; Tomczak, N.; Nallani, M., Chem. Commun. 2011, 47 (10), 2862-2864.

(31) Chiu, H.-C.; Lin, Y.-W.; Huang, Y.-F.; Chuang, C.-K.; Chern, C.-S., Angew. Chem. Int. Ed. 2008, 47 (10), 1875-1878.

(32) Hayward, R. C.; Utada, A. S.; Dan, N.; Weitz, D. A., Langmuir 2006, 22 (10), 4457-4461.

(33) Shum, H. C.; Santanach-Carreras, E.; Kim, J. W.; Ehrlicher, A.; Bibette, J.; Weitz, D. A., J. Am. Chem. Soc. 2011, 133 (12), 4420-4426.

(34) Faivre, M.; Campillo, C.; Pepin-Donat, B.; Viallat, A., Prog. Colloid Polym. Sci. 2006,133, 4144.

(35) Campillo, C.; Pépin-Donat, B.; Viallat, A., Soft Matter 2007, 3 (11), 1421-1427.

(36) Kremer, S.; Campillo, C.; Pepin-Donat, B.; Viallat, A.; Brochard-Wyart, F., Europhysics Letters 2008, 82 (4).

(37) Campillo, C. C.; Schroder, A. P.; Marques, C. M.; Pepin-Donat, B., Soft Matter 2008, 4 (12), 2486-2491.

(38) Campillo, C. C.; Schroder, A. P.; Marques, C. M.; Pépin-Donat, B., Mater. Sci. Eng. C 2009, 29 (2), 393-397.

(39) Kazakov, S.; Kaholek, M.; Teraoka, I.; Levon, K., Macromolecules 2002, 35 (5), 1911-1920.

(40) Saleem, Q.; Liu, B.; Gradinaru, C. C.; MacDonald, P. M., Biomacromolecules 2011, 12 (6), 2364-2374.

(41) Hong, J. S.; Stavis, S. M.; Depaoli Lacerda, S. H.; Locascio, L. E.; Raghavan, S. R.; Gaitan, M., Langmuir 2010, 26 (13), 11581-11588.

(42) Limozin, L.; Sackmann, E., Phys. Rev. Lett. 2002, 89 (16).

(43) Jesorka, A.; Markström, M.; Orwar, O., Langmuir 2005, 21 (4), 1230-1237.

(44) Osinkina, L.; Markström, M.; Orwar, O.; Jesorka, A., Langmuir 2010, 26 (1), 1-4.

(45. Jesorka, A.; Markström, M.; Karlsson, M.; Orwar, O., J. Phys. Chem. B 2005, 109 (31), 1475914763.

(46) Smith, A. M.; Jaime-Fonseca, M. R.; Grover, L. M.; Bakalis, S., J Agric. Food Chem. 2010, 58 (8), 4719-4724.

(47) Hong, J. S.; Vreeland, W. N.; Lacerda, S. H. D.; Locascio, L. E.; Gaitan, M.; Raghavan, S. R., Langmuir 2008, 24 (8), 4092-4096.

(48) Tiwari, S.; Goyal, A. K.; Mishra, N.; Vaidya, B.; Mehta, A.; Dube, D.; Vyas, S. P., Procedia in Vaccinology 2009, 1 (1), 148-163.

(49) Viallat, A.; Dalous, J.; Abkarian, M., Biophys. J.l 2004, 86 (4), 2179-2187.

(50) Mader, M. A.; Vitkova, V.; Abkarian, M.; Viallat, A.; Podgorski, T., Eur. Phys. J. E 2006, 19 (4), 389-397.

(51) Long, M. S.; Jones, C. D.; Helfrich, M. R.; Mangeney-Slavin, L. K.; Keating, C. D., Proc. Natl. Acad. Sci. U.S.A. 2005, 102 (17), 5920-5925.

(52) Lee, H. K.; Soukasene, S.; Jiang, H.; Zhang, S.; Feng, W.; Stupp, S. I., Soft Matter 2008, 4 (5), 962-964.

(53) Lee, J. S.; Zhou, W.; Meng, F.; Zhang, D.; Otto, C.; Feijen, J., J. Controlled Release 2010, 146 (3), 400-408.

(54) Lee, J. S.; Zhou, W.; Zhang, D.; Otto, C.; Feijen, J., J. Controlled Release 2008, 132 (3), e28e29.

(55) Sanson, C.; Schatz, C.; Le Meins, J. F.; Brûlet, A.; Soum, A.; Lecommandoux, S., Langmuir 2010, 26 (4), 2751-2760.

(56) Mabrouk, E.; Cuvelier, D.; Pontani, L. L.; Xu, B.; Lévy, D.; Keller, P.; Brochard-Wyart, F.; Nassoy, P.; Li, M. H., Soft Matter 2009, 5 (9), 1870-1878.

(57) Mabrouk, E.; Cuvelier, D.; Brochard-Wyart, F.; Nassoy, P.; Li, M. H., Proc. Natl. Acad. Sci.U.S.A. 2009, 106 (18), 7294-7298.

(58) Pautot, S.; Frisken, B. J.; Weitz, D. A., Langmuir 2003, 19 (7), 2870-2879.

(59) Mishra, V.; Mahor, S.; Rawat, A.; Dubey, P.; Gupta, P. N.; Singh, P.; Vyas, S. P., Vaccine 2006, 
24 (27-28), 5559-5570.

(60) Ebato, Y.; Kato, Y.; Onishi, H.; Nagai, T.; Machida, Y., Drug Dev. Res. 2003, 58 (3), 253-257.

(61) R.John, E., Trends Biochem. Sci. 2001, 26 (10), 597-604.

(62) Gomez, C. G.; Rinaudo, M.; Villar, M. A., Carbohydr. Polym. 2007, 67 (3), 296-304.

(63) Gomez, C. G.; Pérez Lambrecht, M. V.; Lozano, J. E.; Rinaudo, M.; Villar, M. A., Int. J. Biol. Macromol. 2009, 44 (4), 365-371.

(64) Ochab-Marcinek, A.; Holyst, R., Soft Matter 2011, 7 (16), 7366-7374.

(65) Lekkerkerker, H. N. W.; Tuinier, R.; Lekkerkerker, H., Stability of Colloid-Polymer Mixtures

Colloids and the Depletion Interaction. Springer Berlin / Heidelberg: 2011; Vol. 833, pp 131-175.

(66) Zimmerman, S. B.; Trach, S. O., J. Mol. Biol. 1991, 222 (3), 599-620.

(67) Prouty, M. S.; Schechter, A. N.; Parsegian, V. A., J. Mol. Biol. 1985, 184 (3), 517-528.

(68) Chik, J. K.; Lindberg, U.; Schutt, C. E., J. Mol. Biol. 1996, 263 (4), 607-623.

(69) Le Coeur, C.; Demé, B.; Longeville, S., Phys. Rev. E 2009, 79 (3), 031910.

(70) Le Coeur, C.; Teixeira, J.; Busch, P.; Longeville, S., Phys. Rev. E 2010, 81 (6), 061914.

SYNOPSIS TOC (Word Style “SN_Synopsis_TOC”).

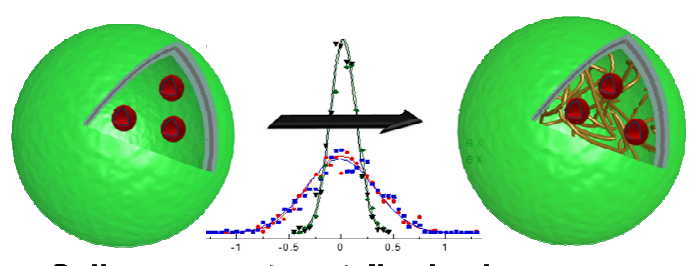

"Gelly" compartmentalized polymersomes:

one step further towards structural cell mimicry 